\title{
Sociodemographic characteristics are associated with prevalence of high-risk waist circumference and high-risk waist-to- height ratio in U.S. adolescents
}

\author{
Felicia J. Setiono ${ }^{1 *}$, Laura A. Guerra ${ }^{2}$, Cindy Leung ${ }^{3}$ and Tashara M. Leak ${ }^{1}$
}

\begin{abstract}
Background: Adiposity status in adolescence is associated with various health outcomes in adulthood. Waist circumference $[\mathrm{WC}]$ and waist-to-height ratio [WHtR] are measures of abdominal obesity and have shown to be valid predictors of future chronic diseases. However, the relationship between sociodemographic characteristics and WC, as well as WHtR in U.S. adolescents remain unclear. Thus, the study aims to examine associations between sociodemographic characteristics and abdominal obesity among a nationally representative sample of U.S. adolescents.

Methods: The sample included 4712 adolescents (12-19years) in the 2007-2016 National Health and Nutrition Examination Survey. Associations between sociodemographic characteristics and abdominal obesity (WC and WHtR) were examined using multiple logistic regression models, adjusted for age, physical activity level, and sedentary activity.

Results: Around $18 \%$ of adolescents had high-risk WC ( $\geq 90$ th percentile) and $34 \%$ had high-risk WHtR ( $\geq 0.5)$. Females had higher odds of high-risk WHtR compared to males $(\mathrm{OR}=1.46,95 \% \mathrm{Cl}=1.23-1.72)$. Mexican American adolescents had higher odds of high-risk WHtR compared to non-Hispanic White $(\mathrm{OR}=1.66,95 \% \mathrm{Cl}=1.24-2.20)$, non-Hispanic Black $(\mathrm{OR}=1.73,95 \% \mathrm{Cl}=1.26-2.36)$, and other race/multi-racial adolescents $(\mathrm{OR}=1.84,95 \% \mathrm{Cl}=1.21$ 2.80). When their parent were college graduates, adolescents had lower odds for high-risk WC compared to when the parent had some college education $(\mathrm{OR}=0.68,95 \% \mathrm{Cl}=0.49-0.93)$ or a high school degree or less $(\mathrm{OR}=0.70$, $95 \% \mathrm{Cl}=0.51-0.97$ ). Similar associations were seen between parental education level and high-risk WHtR, as well as between household income and high-risk WHtR.

Conclusions: Measures of abdominal obesity should be considered to assess burden of adiposity, especially among female adolescents, adolescents from racial/ethnic minority and low socioeconomic status backgrounds. Additionally, future health interventions should consider including changes in WC and WHtR to measure the impact of these interventions.
\end{abstract}

Keywords: Waist circumference, Waist-to-height ratio, Adolescents, Abdominal obesity, Adiposity

\footnotetext{
* Correspondence: fjs66@cornell.edu

${ }^{1}$ Division of Nutritional Sciences, Cornell University, Ithaca, NY 14853, USA

Full list of author information is available at the end of the article
}

(c) The Author(s). 2021 Open Access This article is licensed under a Creative Commons Attribution 4.0 International License, which permits use, sharing, adaptation, distribution and reproduction in any medium or format, as long as you give appropriate credit to the original author(s) and the source, provide a link to the Creative Commons licence, and indicate if changes were made. The images or other third party material in this article are included in the article's Creative Commons licence, unless indicated otherwise in a credit line to the material. If material is not included in the article's Creative Commons licence and your intended use is not permitted by statutory regulation or exceeds the permitted use, you will need to obtain permission directly from the copyright holder. To view a copy of this licence, visit http://creativecommons.org/licenses/by/4.0/. The Creative Commons Public Domain Dedication waiver (http://creativecommons.org/publicdomain/zero/1.0/) applies to the data made available in this article, unless otherwise stated in a credit line to the data. 


\section{Background}

Obesity during adolescence is a risk factor for various chronic diseases in adulthood [1-3]. The most common measure of obesity in adolescents is body mass index (BMI), where a BMI exceeding the age- and sex- specific 95th percentile (equivalent to BMI z-score of 1.64) indicates obesity (based on the 2000 Centers for Disease Control and Prevention (CDC) BMI-for-age growth charts) $[4,5]$. In the U.S., adolescents (12-19 years) experience a greater prevalence of obesity compared to younger children. Using data from National Health and Nutrition Examination Survey (NHANES) from 2013 to 2016, Ogden et al. reported the prevalence of obesity in adolescents is $20.6 \%(95 \% \mathrm{CI}=17.6-23.8)$ [6]. Additionally, it is known that sociodemographic characteristics are associated with obesity prevalence; adolescents from racial/ethnic minority and low socioeconomic status (SES) households experience higher prevalence of obesity than their non-Hispanic white and higher SES counterparts [6-9].

While BMI is often used as an indicator for adiposity, it is often criticized because it does not differentiate between lean mass and fat [10]. In contrast, waist circumference (WC) and waist-to-height ratio (WHtR) are measures of abdominal obesity and are shown to be better predictors of cardiovascular disease risk factors, type 2 diabetes, and metabolic syndrome [11-16]. Despite the value of these measures, studies examining adolescents' WC and WHtR are sparse and less is known about sociodemographic disparities in WC and WHtR among U.S. adolescents. Using 2003-2012 NHANES data, Xi and colleagues found that female adolescents have a higher prevalence of high-risk WC and WHtR compared to males, and that Mexican American adolescents have a significantly higher prevalence of high-risk WC and WHtR compared to their non-Hispanic white counterparts [11]. Research suggests that higher parental education level and household income are associated with lower prevalence of BMI among U.S. adolescents $[6,17$, 18 ], but the relationship between these SES factors and WC, as well as WHtR, among U.S. adolescents in recent years remains unknown. Using more recently available data from 2007 to 16 NHANES, the overall aim of this study was to examine, 1) the prevalence of high-risk WC ( $\geq 90$ th percentile) and WHtR $(\geq 0.5)$, and 2 ) associations between sociodemographic characteristics and high-risk WC and WHtR, among a nationally representative sample of U.S. adolescents (12-19 years).

\section{Methods}

\section{Study design and population}

The NHANES is a complex, multistage probability cross-sectional study of a nationally representative sample of U.S. civilian, noninstitutionalized populations [19].
The National Center for Health Statistics within CDC conducts the NHANES in two-year cycles. For the current study, five NHANES cycles, 2007-2016, were included in the analyses. A total of 5795 adolescents (1219 years) participated in NHANES 2007-2016 and 4712 were included in the analyses with complete sociodemographic, WC, and WHtR data. Adolescents 12-17 years provided assent and their parents provided consent, while adolescents 18-19 years provided informed consent and did not require parental consent. All of the data collection processes were approved by the National Center for Health Statistics Research Ethics Review Board (protocol \#2005-06 for years 2007-2010, and protocol \#2011-17 for years 2011-2016).

\section{Measures}

Waist circumference (WC) and waist-to-height ratio (WHtR)

Anthropometric measures were collected in the Mobile Examination Center (MEC) by trained health technicians. Height $(\mathrm{cm})$ and WC $(\mathrm{cm})$ measurements were collected using standard protocols and equipment [20]. Age and sex-specific WC percentiles were calculated as previously done in other studies [11] and $\geq 90$ th percentile was considered high risk [21]. Lastly, WHtR was calculated by taking WC $(\mathrm{cm})$ and dividing it by height (cm). A WHtR of $\geq 0.5$ was considered high risk [11]. Adolescents with high-risk WC or WHtR were considered to have abdominal obesity.

\section{Sociodemographic characteristics}

The demographic questionnaire was administered in the home by trained interviewers. Adolescents aged 16-19 were interviewed directly, whereas those aged 12-15 had a proxy to respond to questions when the child was unable to answer. Adolescents self-reported their sex (male or female) and race/ethnicity. Race/ethnicity categories reported in this study included non-Hispanic $(\mathrm{NH})$ white, NH black, Mexican American, other Hispanics, and other race/multi-racial. Starting in 2011-2012 survey cycle, the $\mathrm{NH}$ Asian category was added to the list of NHANES racial/ethnic categories. For this study, NH Asian was included in the other race/multi-racial group in order to maintain consistency throughout the five data cycles.

Two measures are used as proxies for household SES. The first is the education level of the household reference person. This data was collected and was categorized as follows: high school/general education diploma (GED) or less (included those with $<9$ th grade, 9-11th grade education, high school/GED or equivalent degrees), some college (those with some college or Associate degrees), and college graduates or above. The household reference person is the person who owns or rents the house and is most likely the parent. Thus, this 
variable will be henceforth referred to as parental education level. The second measure is the poverty to income ratio (PIR), which was calculated by dividing family income by the poverty threshold specific to the survey year, also accounting for family size. The PIR falls within three categories; low-income (PIR $\leq 1.3$ ), middle-income $(\mathrm{PIR}>1.3-3.5)$, and high-income $(\mathrm{PIR}>3.5)$ [17].

\section{Physical activity}

Adolescents aged 12 to 15 years completed the physical activity questionnaire at the MEC, whereas adolescents 16 years and older completed the questionnaire at home prior to visiting the MEC. In this study, physical activity level (PAL) was assessed by two questions, as previously done in another study [22]; "In a typical week do you do any vigorous-intensity sports, fitness, or recreational activities that cause large increases in breathing or heart rate like running or basketball for at least 10 minutes continuously?", and "In a typical week, do you do any moderate-intensity sports, fitness, or recreational activities that cause a small increase in breathing or heart rate such as brisk walking, bicycling, swimming, or volleyball for at least 10 minutes continuously?". Adolescents who reported 'yes' to both questions were categorized as having a high PAL, those who reported 'yes' to only one question were considered to have a medium PAL, and those who said no to both questions were considered to have a low PAL.

\section{Sedentary activity}

Sedentary activity was asked during the physical activity questionnaire and was analyzed from the question "How much time do you usually spend sitting on a typical day?", which may include time spent sitting at a desk, traveling in a car or bus, reading, playing cards, watching television or using a computer. The amount of sedentary time was analyzed as a continuous variable and reported in minutes/day.

\section{Statistical analyses}

Survey weights, strata, and clustering variables were used to account for the complex, multistage probability sampling design used in NHANES, according to the analytic guidelines released by National Center for Health Statistics and CDC [23, 24]. Examination weights were used to create 10 -year weights (i.e., 5 cycles) for all analyses. Continuous variables were described using weighted means and categorical variables were summarized with weighted proportions. Standard errors were estimated with Taylor series linearization. The prevalence of highrisk WC and WHtR by survey year and sociodemographic characteristics categories were calculated as percentages with $95 \%$ confidence interval (CI). A logistic regression model was conducted to analyze trend of high-risk WC and WHtR across survey years.

Associations between adiposity measures and sociodemographic characteristics (sex, race/ethnicity, parental education level, and PIR) were analyzed using multiple logistic regression models, adjusted for all sociodemographic characteristics, along with age, PAL and sedentary activity. Multicollinearity was assessed by calculating the variance inflation factor. No variables were found to be collinear between one another (VIF $\sim 1$ for all variables). Significance level was set at $p \leq 0.05$. For covariates with multiple categories, odds ratios of abdominal obesity outcomes between the different categories were calculated, adjusted using a Bonferroni correction. $P$ values for overall trend for parental education level and PIR were also calculated. All analyses were conducted in STATA 15 (StataCorp, College Station, TX).

\section{Results}

Unweighted sample size and weighted means and proportions for individual and household characteristics of the study sample $(n=4712)$ are presented in Table 1 . The weighted mean age of the sampled adolescents was 15.5 years. About half of the adolescents were male $(50.9 \%)$ and half were female $(49.1 \%)$. The majority of the adolescents were $\mathrm{NH}$ white $(58.6 \%)$, followed by $\mathrm{NH}$ black (13.9\%), Mexican American (13.1\%), other race/ multi-racial (7.7\%) and other Hispanic (6.7\%). About $41.0 \%$ of the adolescents' parent had a high school degree or less, while $31.9 \%$ reported to have some college education, and $27.1 \%$ reported to be college graduates. Approximately one-third (30.5\%) of the adolescents reside in a low-income household (PIR $\leq 1.3$ ), 37.4\% reside in a middle-income household (PIR >1.3-3.5), and $32.2 \%$ reside in a high-income household (PIR $>3.5$ ). Based on the physical activity categories, $18.9 \%$ of adolescents had low PAL, 43.1\% had medium PAL, and $38.0 \%$ have high PAL. On average, adolescents spend $484.8 \mathrm{~min}$ (a little over $8 \mathrm{~h}$ ) of sedentary activities each day. About $18 \%$ of adolescents had high-risk WC ( $\geq 90$ th percentile) and $35.5 \%$ of adolescents had high-risk WHtR $(\geq 0.5)$.

The prevalence of high-risk WC and high-risk WHtR are presented across survey cycles and sociodemographic characteristics in Table 2. The prevalence of high-risk WC ranged from 14.1 to $22.8 \%$ and from 30.7 to $49.8 \%$ for high-risk WHtR across the race/ethnicity categories. The prevalence of high-risk WC and WHtR are lower with higher levels of parental education and PIR. There were no significant trends in prevalence of high-risk WC and high-risk WHtR over survey years.

Associations between sociodemographic characteristics and high-risk WC and WHtR are presented in Table 3. 
Table 1 Individual and household characteristics of adolescents (12-19years) in NHANES 2007-2016 with examination data ( $n=$ 4712)

\begin{tabular}{|c|c|c|}
\hline Characteristics & Unweighted $\mathrm{N}^{\mathrm{a}}$ & Weighted \% (SE) ${ }^{\mathrm{a}}$ \\
\hline Age, mean (SD) & $15.47(1.98)$ & $15.49(0.04)$ \\
\hline \multicolumn{3}{|l|}{ Sex } \\
\hline Male & 2433 & $50.9 \%(0.92)$ \\
\hline Female & 2279 & $49.1 \%(0.92)$ \\
\hline \multicolumn{3}{|l|}{ Race/ethnicity } \\
\hline NH White & 1390 & $58.6 \%(2.15)$ \\
\hline NH Black & 1171 & $13.9 \%(1.18)$ \\
\hline Mexican American & 1043 & $13.1 \%(1.27)$ \\
\hline Other Hispanic & 538 & $6.7 \%(0.70)$ \\
\hline Other race/multi-racial & 570 & $7.7 \%(0.64)$ \\
\hline \multicolumn{3}{|l|}{ Parental education ${ }^{b}$} \\
\hline High school/GED or less & 2339 & $41.0 \%(1.71)$ \\
\hline Some college & 1404 & $31.9 \%(1.16)$ \\
\hline College graduate & 969 & $27.1 \%(1.57)$ \\
\hline \multicolumn{3}{|l|}{ Poverty to income ratio ${ }^{b}$} \\
\hline Low-income $(\mathrm{PIR} \leq 1.3)$ & 1996 & $30.5 \%(1.52)$ \\
\hline Middle-income (PIR > 1.3-3.5) & 1683 & $37.4 \%(1.38)$ \\
\hline High-income (PIR > 3.5) & 1033 & $32.2 \%(1.67)$ \\
\hline \multicolumn{3}{|l|}{ Physical activity level ${ }^{b}$} \\
\hline Low & 1008 & $18.9 \%(0.75)$ \\
\hline Medium & 2074 & $43.1 \%(1.00)$ \\
\hline High & 1630 & $38.0 \%(1.06)$ \\
\hline Sedentary activity minutes (mean (SD)) & $485.76(174.47)$ & $484.8(4.38)$ \\
\hline High-risk waist circumference ${ }^{b}$ & 8555 & $17.7 \%(0.84)$ \\
\hline High-risk waist-to-height ratio ${ }^{c}$ & 1785 & $35.5 \%(1.03)$ \\
\hline
\end{tabular}

NHANES National Health and Nutrition Examination Survey, BMI Body mass index, SE Standard error, SD Standard deviation, GED General education diploma, PIR Poverty to income ratio

a Unweighted N (\%) represent raw data, weighted \% (SE) used 2007-2016 survey weights. Units are as specified unless otherwise stated

${ }^{\mathrm{b}}$ High-risk waist circumference defined as greater than or equal to the gender- and age-specific 90th percentile

'High-risk waist-to-height ratio defined as $\geq 0.5$

There was a significant linear trend in high-risk WC by parental education level $(p=0.004)$. When the parent had a college degree, adolescents were less likely to have a high-risk WC $(\mathrm{OR}=0.57,95 \% \mathrm{CI}=0.37-0.87, p=$ 0.006) than when parent had a high school degree or less. Adolescents whose parent had a college degree were also significantly less likely to have high-risk WC compared to those whose parent had some college education $(\mathrm{OR}=0.62,95 \% \mathrm{CI}=0.43-0.89, p=0.006)$. While there were no significant differences in the likelihood of having high-risk WC by household income categories, there was overall, a significant linear trend of lower odds for high-risk WC as household PIR increases $(p=0.04)$ (Table 3). There was no association between other sociodemographic characteristics and high-risk WC.

Regarding WHtR, females were more likely to have a high-risk WHtR compared to males $(\mathrm{OR}=1.46,95 \% \mathrm{CI}=$
1.23-1.72, $p<0.001)$. Mexican American adolescents had higher odds for high-risk WHtR compared to $\mathrm{NH}$ white $(\mathrm{OR}=1.66,95 \% \mathrm{CI}=1.24-2.20, \mathrm{p}<0.001), \quad \mathrm{NH}$ black $(\mathrm{OR}=1.73, \quad 95 \% \mathrm{CI}=1.26-2.36, \quad p<0.001)$ and other race/multi-racial adolescents $(\mathrm{OR}=1.84,95 \% \mathrm{CI}=$ 1.21-2.80, $p=0.001)$. There were significant linear trends in high-risk WHtR by parental education level $(p=0.01)$ and household PIR $(p<0.001)$. Adolescents with parent who had a college degree had significantly lower odds for high-risk WHtR than adolescents whose parent had some college education $(\mathrm{OR}=0.68,95 \% \mathrm{CI}=$ $0.49-0.93, p=0.01)$ and had a high school degree or less $(\mathrm{OR}=0.70,95 \% \mathrm{CI}=0.51-0.97, p=0.03)$. For PIR, odds for high-risk WHtR was significantly lower in adolescents living in high-income households than in adolescents living in low-income households $(\mathrm{OR}=0.58$, $95 \% \mathrm{CI}=0.43-0.78, p<0.001)$ and adolescents residing 
Table 2 Prevalence of high-risk waist circumference and high-risk waist-to-height ratio in adolescents by sociodemographic characteristics $(n=4712)$

\begin{tabular}{|c|c|c|}
\hline & Prevalence, \% (95\% Cl) & \\
\hline & High-risk waist circumference ( $\geq 90$ th percentile) & High-risk waist-to-height ratio $(\geq 0.5)$ \\
\hline Survey year & & \\
\hline $2007-08$ & $17.5(14.5-20.9)$ & $34.7(30.5-39.1)$ \\
\hline 2009-10 & $15.2(13.2-17.4)$ & $31.3(28.4-34.4)$ \\
\hline $2011-12$ & $18.1(14.2-22.7)$ & $37.0(33.9-40.2)$ \\
\hline $2013-14$ & $18.1(14.8-22.1)$ & $36.4(30.9-42.2)$ \\
\hline $2015-16$ & $19.3(15.0-24.6)$ & $37.6(32.1-43.5)$ \\
\hline Sex & & \\
\hline Male & $16.8(14.9-18.9)$ & $30.8(28.3-33.5)$ \\
\hline Female & $18.6(16.4-21.0)$ & $40.2(37.4-43.1)$ \\
\hline Race/ethnicity & & \\
\hline NH White & $16.6(14.4-19.0)$ & $32.0(29.0-35.1)$ \\
\hline $\mathrm{NH}$ Black & $18.8(16.1-21.7)$ & $35.4(32.1-38.9)$ \\
\hline Mexican American & $22.8(20.2-25.7)$ & $49.8(46.5-53.0)$ \\
\hline Other Hispanic & $18.9(15.1-23.3)$ & $43.5(39.1-48.0)$ \\
\hline Other/multi-racial & $14.1(10.6-18.4)$ & $30.7(25.6-36.3)$ \\
\hline Parental education & & \\
\hline High school/GED or less & $21.3(18.7-24.2)$ & $41.3(38.8-43.8)$ \\
\hline Some college & $18.6(16.5-21.0)$ & $37.2(33.8-40.7)$ \\
\hline College graduate & $11.0(8.7-13.9)$ & $24.5(21.1-28.3)$ \\
\hline Poverty to income ratio & & \\
\hline Low-income $(P I R \leq 1.3)$ & $21.6(19.2-24.1)$ & $43.9(41.2-46.6)$ \\
\hline Middle-income (PIR > 1.3-3.5) & $18.8(16.2-21.6)$ & $37.6(34.1-41.3)$ \\
\hline High-income (PIR > 3.5) & $12.7(10.3-15.6)$ & $24.9(21.7-28.5)$ \\
\hline
\end{tabular}

NHANES National Health and Nutrition Examination Survey, Cl Confidence interval, NH Non-Hispanic GED General education diploma, PIR Poverty to income ratio

in middle-income household $(\mathrm{OR}=0.68,95 \% \mathrm{CI}=0.48-$ $0.96, p=0.022$ ).

\section{Discussion}

In the U.S., a large percentage of adolescents have abdominal obesity. However, there is limited information on the association between various sociodemographic characteristics and WC, as well as WHtR, among U.S. adolescents. Understanding sociodemographic disparities in abdominal obesity is important as high WC and WHtR in adolescents have been shown to predict future adverse health outcomes [12-16]. This study described the prevalence of $\mathrm{WC}$ and $\mathrm{WHtR}$, as well as associations between several sociodemographic characteristics and $\mathrm{WC}$ and $\mathrm{WHtR}$, among a nationally representative sample of U.S. adolescents.

In the current study, female adolescents had greater odds for a high-risk WHtR compared to males, but there was no association between sex and WC. By contrast, Xi et al. (2014) found female children and adolescents (219 years) to be at greater risk for both WHtR and WC than males [11]. This discrepancy could be due to the age differences in the sample used by the current study (12-19 years) and that of Xi et al.'s. (2-19 years). Additionally, the current study used data from 2007 to 2016, whereas Xi et al.'s study used data from 2003 to 2012. Another possible explanation for the lack of association between sex and WC is that fat distribution in male adolescents becomes more centered in the abdominal region during late puberty (age 14), whereas for females, fat distribution remains in the gynoid pattern throughout puberty [25]. Moreover, there is evidence that male adolescents typically exceed female adolescents in height by age 14 and they continue to grow until they reach their peak height [26]. Due to these factors, male adolescents' WHtR on average, would be smaller than that for female adolescents given similar WC. These developmental changes may explain why differences in WHtR by sex, but not WC, were observed in the current study.

Mexican American adolescents are significantly more likely to have high-risk WHtR, compared to $\mathrm{NH}$ white and black, and other race/multi-racial adolescents. 
Table 3 Odds ratios of high-risk waist circumference and high-risk waist-to-height ratio in adolescents by sociodemographic characteristics $(n=4712)$

\begin{tabular}{|c|c|c|}
\hline & \multicolumn{2}{|l|}{ Odds ratios $(95 \% \mathrm{Cl})$} \\
\hline & High-risk waist circumference ( $\geq 90$ th percentile) & High-risk waist-to-height ratio $(\geq 0.5)$ \\
\hline \multicolumn{3}{|l|}{ Sex } \\
\hline Male & $\operatorname{Ref}^{a}$ & $\operatorname{Ref}^{a}$ \\
\hline Female & $1.05(0.87-1.28)^{\mathrm{a}}$ & $1.46(1.23-1.72)^{\mathrm{b}}$ \\
\hline \multicolumn{3}{|l|}{ Race and ethnicity } \\
\hline NH white & $\operatorname{Ref}^{a}$ & $\operatorname{Ref}^{a}$ \\
\hline $\mathrm{NH}$ black & $0.96(0.67-1.38)^{\mathrm{a}}$ & $0.96(0.69-1.32)^{\mathrm{a}}$ \\
\hline Mexican American & $1.15(0.80-1.63)^{a}$ & $1.66(1.24-2.20)^{b}$ \\
\hline Other Hispanic & $0.94(0.60-1.48)^{\mathrm{a}}$ & $1.33(0.95-1.87)^{\mathrm{ab}}$ \\
\hline Other/multi-racial & $0.76(0.45-1.28)^{\mathrm{a}}$ & $0.90(0.58-1.41)^{\mathrm{a}}$ \\
\hline \multicolumn{3}{|l|}{ Parental education } \\
\hline High school/GED or less & $\operatorname{Ref}^{a}$ & $\operatorname{Ref}^{a}$ \\
\hline Some college & $0.92(0.66-1.28)^{\mathrm{a}}$ & $1.03(0.80-1.33)^{\mathrm{a}}$ \\
\hline College graduate & $0.57(0.37-0.87)^{\mathrm{b}}$ & $0.70(0.51-0.97)^{b}$ \\
\hline$P$ value ${ }^{c}$ & 0.004 & 0.01 \\
\hline \multicolumn{3}{|l|}{ Poverty to income ratio } \\
\hline Low-income $(\mathrm{PIR} \leq 1.3)$ & $\operatorname{Ref}^{a}$ & $\operatorname{Ref}^{a}$ \\
\hline Middle-income (PIR > 1.3-3.5) & $0.92(0.67-1.26)^{a}$ & $0.86(0.66-1.11)^{\mathrm{a}}$ \\
\hline High-income (PIR > 3.5) & $0.72(0.48-1.07)^{\mathrm{a}}$ & $0.58(0.43-0.78)^{b}$ \\
\hline$P$ value ${ }^{c}$ & 0.04 & $<0.001$ \\
\hline
\end{tabular}

NHANES National Health and Nutrition Examination Survey, Cl Confidence interval, Ref Reference category, NH Non-Hispanic, GED General education diploma Multiple logistic regression models were adjusted for age, physical activity level, and sedentary activity. Values are odds ratios (95\% $\mathrm{Cl}$ ) between a level of a variable compared to the reference category

a, bValues with different subscripts are significantly different at $P \leq 0.05$ within each variable after Bonferroni correction

${ }^{c} P$ value for trend used when categories within a variable is ordered, significance level is set at $p \leq 0.05$

Higher prevalence of abdominal obesity as measured by WHtR in Mexican American adolescents compared to $\mathrm{NH}$ white adolescents has been reported previously using NHANES data from 2003 to 2012 [11]. Although, comparisons of prevalence of high-risk WHtR between Mexican American adolescents and adolescents of other race/ethnic identities have never been evaluated.

It is known that WHtR distinguishes between lean mass and fat and has been suggested to be a better predictor of adiposity compared to BMI [27]. A couple of studies have also argued that BMI is less reliable when used to examine differences in adiposity between racial and ethnic groups since the relationship between body fat composition and BMI differs across ethnic groups $[28,29]$. Thus, associations between sociodemographic characteristics with abdominal obesity and BMI were not consistent. In the current study, likelihood of having high-risk WHtR in NH black adolescents were not significantly different than that in adolescents of any other race/ethnic identities. On the other hand, studies focusing on excess BMI in adolescents commonly report that obesity prevalence in $\mathrm{NH}$ black adolescents are significantly higher than in $\mathrm{NH}$ white adolescents $[6,7]$.
In the current study, there were no significant differences in likelihood of having high-risk WC by race/ethnicity. In contrast, previous studies that analyzed NHAN ES data from 1988 to 2002 and 2003 to 2012 found those who identified as Mexican Americans or Hispanics were more likely to have high-risk WC compared to $\mathrm{NH}$ white and $\mathrm{NH}$ black youth $[11,30]$. The data used in the current study includes the most recent NHANES cycle (2015-2016 cycle). Thus, it is possible that associations between race/ethnicity and high-risk WC have changed over the years. Additionally, unlike the previous studies, the current study used Bonferroni correction to adjust for multiple comparisons within the different race/ethnic categories which resulted in larger confidence intervals for the odds ratios calculated in the models. Another study, by Tybor et al. (2010), found NH black female adolescents having significantly higher WC than white female adolescents [31]. Tybor et al., however, focused only on female adolescents rather than both male and female adolescents, as done in the current study [31].

To the authors' knowledge, this is the first study to report that both high-risk WC and WHtR in U.S. adolescents are associated with parental education (a proxy for 
household SES). For both WC and WHtR, the odds of abdominal obesity were significantly lower in adolescents whose parent are college graduates, compared to parent who have some college education, indicating that higher educational attainment in parent is related to the health status of adolescents. This result is consistent with studies that examined associations between SES and BMI, which have shown that obesity prevalence among U.S. adolescents is inversely associated with parental education level $[6,17]$. For example, Ogden and colleagues (2018) found that U.S. children and adolescents (2-19 years) with parent with a high school degree or less were significantly more likely to be obese compared to youth whose parent graduated from college, $22 \%$ and $12 \%$ respectively [6]. Furthermore, this result also corroborates analyses of the Healthy Lifestyle in Europe by Nutrition in Adolescence (HELENA) study, where parental education level was inversely associated with WC and WHtR [32]. It has been suggested that education level is associated with dietary intake/quality as those with higher education may have access to greater nutrition knowledge, which also better allows for incorporation of such knowledge into their dietary habits [33].

In the current study, likelihood of high-risk WC did not differ significantly across PIR categories. This result aligns with analyses of the HELENA study in Europe which found no association between affluence (measured by Family Affluence Scale) and WC [32]. By contrast, this study found that odds of having high-risk WHtR is significantly lower in adolescents living in high-income households (PIR > 3.5) compared to adolescents living in middle-income and low-income households. Similarly, Ali et al. (2011), using data from NHANES 1998-2008, reported that prevalence of high-risk WHtR in children and adolescents was inversely related to household income status [34]. Other NHANES studies have also found that BMI is significantly lower in adolescents living in high-income households compared to low-income households $[17,18]$.

While parental education level and PIR are both indicators of SES, this study found that the association between the two measures and abdominal obesity are different. Previous research has shown parental education level may be a better indicator for an individual's weight status and other chronic conditions [17, 35]. Despite the differences, this study revealed a general trend where adolescents from low SES households have a greater risk for abdominal obesity. One possible explanation of the relationship between SES and abdominal obesity is that individuals residing in higher income neighborhoods may have more access to recreation and pedestrian/biking facilities, and thus higher levels of physical activity compared to individuals in low-income neighborhoods [36]. Individuals living in higher-income areas also have more access to healthier foods [37]. In fact, neighborhoods' walkability, shopping facilities, and existence of parks/playgrounds and SES have been found to be associated with WC in children and adolescents with severe obesity [38].

This study is not without limitations. In NHANES, the sample size for some demographic subgroups are small, which may result in inaccurate estimates. To overcome this, the study combined multiple data cycles (2007-2016) to obtain a higher sample size for specific demographic subgroups, such as other Hispanic and other race/multi-racial adolescents. They were included in the study as separate categories since there is little data on the adiposity status for adolescents in these groups otherwise. The study used cross-sectional data, thus no conclusions regarding causality between sociodemographic characteristics and abdominal obesity can be made. While multiple statistical tests were conducted, associations reported in the study were corrected to account for multiple comparisons, increasing the robustness of the statistical analyses.

Categories for PAL used may not accurately estimate how active an adolescent is as it did not distinguish the types and actual duration of physical activity that adolescents engage in. Although the Metabolic Equivalent of Task (MET) scores can be used to measure PAL [39], information needed to calculate METs score had a significant proportion of missing data and was deemed insufficient for analyses. This study did not include information on nutritional intake and pubertal development which could affect adiposity and fat distribution in adolescents. Additionally, pregnant adolescents were not excluded from the study even though pregnancy status would impact adiposity status. Pregnancy status in NHANES 2007-16 were not released for individuals below the age of 20 due to disclosure risks [40]. However, according to NHANES data documentation, the proportion of pregnant adolescents aged 12-19 years old were estimated to be very low $(1-2 \%)$ [40], thus the inclusion of pregnant adolescents likely had little impact on the results.

Despite these limitations, this study has several noteworthy strengths. As discussed above, the current study is one of the first studies to explore the association of household SES and both WC and WHtR among U.S. adolescents in recent years. Moreover, data from NHANES is nationally representative, thus results from this study are generalizable to U.S. adolescents. Anthropometrics measurements were collected by trained staff using standard protocols [20] and thus, estimates of WC and WHtR presented in the study were less prone to measurement error. 


\section{Conclusion}

This study addresses a critical gap in the literature by revealing that sociodemographic characteristics (i.e., sex, racial/ethnic identities, parental education level, and PIR) are associated with high-risk WC and WHtR among U.S. adolescents. Many studies that have examined associations between sociodemographic characteristics and adiposity use BMI as a measure of adiposity [6, $8,41]$. However, assessing only BMI may not fully capture the burden of adiposity in adolescents. This is important as disparities in chronic disease prevalence persist into adulthood; with rates reported to be significantly higher in racial/ethnic minority and low-income adults [42-44]. Future interventions for adolescents should prioritize those who are most at-risk for abdominal obesity, namely female adolescents, as well as adolescents from racial/ethnic minority and low socioeconomic status backgrounds. Additionally, such interventions should consider including changes in WC and WHtR as a measure of impact of these interventions, rather than just BMI.

\begin{abstract}
Abbreviations
WC: Waist circumference; WHtR: Waist-to-height ratio; BMl: Body mass index; CDC: Centers for Disease Control and Prevention; SES: Socioeconomic status; NHANES: National Health and Nutrition Examination Survey; $\mathrm{NH}$ : NonHispanic; GED: General education diploma; PIR: Poverty to income ratio; MEC: Mobile examination center; PAL: Physical activity level; Cl: Confidence interval; SD: Standard deviation; SE: Standard error; HELENA: Healthy Lifestyle in Europe by Nutrition in Adolescence; MET: Metabolic Equivalent of Task
\end{abstract}

\section{Acknowledgements}

We would like to acknowledge Francoise Vermeylen for her input on the statistical analyses used in this manuscript

\section{Authors' contributions}

FJS analyzed and interpreted data, conducted literature searches, and had primary responsibility of writing the manuscript. $L A G$ and $C L$ analyzed data and reviewed and revised the manuscript. TML interpreted data and contributed significantly to editing the manuscript. All authors read and approved the final manuscript.

\section{Funding}

No funding was received for this manuscript.

\section{Availability of data and materials}

All data presented in the manuscript are publicly available through the Centers for Disease Control and Prevention site at https://wwwn.cdc.gov/ nchs/nhanes/default.aspx [45]. Additionally, STATA software was used. Operating system: Platform independent, requirements: STATAIC 14 or higher, license: individual, any restrictions to use by non-academics: license needed.

\section{Declarations}

\section{Ethics approval and consent to participate}

Adolescents 12-17 years provided assent and informed consent was obtained from their parents. Adolescents 18-19 years provided informed consent and did not require parental consent. All of the data collection processes were approved by the National Center for Health Statistics Research Ethics Review Board (protocol \#2005-06 and protocol \#2011-17). All research methods were carried out in accordance with relevant guidelines and regulations.
Consent for publication

Not applicable.

\section{Competing interests}

The authors declare that they have no competing interests.

\section{Author details}

1Division of Nutritional Sciences, Cornell University, Ithaca, NY 14853, USA.

${ }^{2}$ Department of Health \& Behavior Studies, Teachers College, Columbia

University, New York, NY, USA. ${ }^{3}$ Department of Nutritional Sciences,

University of Michigan School of Public Health, Ann Arbor, MI, USA.

Received: 4 November 2020 Accepted: 16 April 2021

Published online: 03 May 2021

\section{References}

1. Freedman DS, Sherry B. The validity of BMI as an indicator of body fatness and risk among children. Pediatrics. 2009;124(1):S23.

2. Daniels SR, Arnett DK, Eckel RH, Gidding SS, Hayman LL, Kumanyika S, et al. Overweight in children and adolescents: pathophysiology, consequences, prevention, and treatment. Circulation. 2005;111(15):1999-2012

3. Ebbiling CB, Pawluk D, Ludwig DS. Childhood obesity public-health crisis, common sense cure. Lancet. 2012;360:473-82 Available from: https://www. sciencedirect.com/science/article/pii/S0140673602096782?via\%3Dihub.

4. Ogden $\mathrm{CL}$, Carroll MD, Flegal KM. High body mass index for age among US children and adolescents, 2003-2006. J Am Med Assoc. 2008;299(20):2401-5.

5. CDC. Data Table of BMl-for-age Charts. CDC Growth Charts. 2001 [cited 2020 Apr 3]. Available from: https://www.cdc.gov/growthcharts/html_cha rts/bmiagerev.htm

6. Ogden CL, Fryar CD, Hales CM, Carroll MD, Aoki Y, Freedman DS. Differences in obesity prevalence by demographics and urbanization in US children and adolescents, 2013-2016. J Am Med Assoc. 2018;319(23):2410-8.

7. Anderson PM, Butcher KF, Schanzenbach DW. Understanding recent trends in childhood obesity in the United States. Econ Hum Biol. 2019;34:16-25. https://doi.org/10.1016/j.ehb.2019.02.002.

8. Ogden $\mathrm{CL}$, Carroll MD, Lawman HG, Fryar CD, Kruszon-Moran D, Kit BK, et al. Trends in Obesity Prevalence Among Children and Adolescents in the United States, 1988-1994 Through 2013-2014. JAMA. 2016;315(21):2292 Available from: http://jama.jamanetwork.com/article.aspx?doi=10.1001/ja ma.2016.6361.

9. Skinner AC, Ravanbakht SN, Skelton JA, Perrin EM, Armstrong SC. Prevalence of Obesity and Severe Obesity in US Children, 1999-2016. Pediatrics. 2018; 141(3):e20173459 Available from: http://pediatrics.aappublications.org/ lookup/doi/10.1542/peds.2017-3459.

10. Yajnik CS, Yudkin JS. Clinical picture the Y-Y paradox. Lancet. 2004;363:163.

11. Xi B, Mi J, Zhao M, Zhang T, Jia C, Li J, et al. Trends in abdominal obesity among US children and adolescents. Pediatrics. 2014;134(2):e334.

12. Savva SC, Tornaritis M, Savva ME, Kourides Y, Panagi A, Silikiotou N, et al. Waist circumference and waist-to-height ratio are better predictors of cardiovascular disease risk factors in children than body mass index. Int J Obes. 2000;24(11):1453-8.

13. Spolidoro JV, Pitrez Filho ML, Vargas LT, Santana JC, Pitrez E, Hauschild JA, et al. Waist circumference in children and adolescents correlate with metabolic syndrome and fat deposits in young adults. Clin Nutr. 2013;32(1): 93-7. https://doi.org/10.1016/j.clnu.2012.05.020.

14. Saydah S, Bullard KM, Imperatore G, Geiss L, Gregg EW. Cardiometabolic risk factors among US adolescents and young adults and risk of early mortality. Pediatr Int. 2013;131(3):e679-86 Available from: http://pediatrics.aappublica tions.org/cgi/doi/10.1542/peds.2012-2583.

15. Lee CMY, Huxley RR, Wildman RP, Woodward M. Indices of abdominal obesity are better discriminators of cardiovascular risk factors than BMI: a meta-analysis. J Clin Epidemiol. 2008;61(7):646-53.

16. Wicklow BA, Becker A, Chateau D, Palmer K, Kozyrskij A, Sellers EAC. Comparison of anthropometric measurements in children to predict metabolic syndrome in adolescence: analysis of prospective cohort data. Int J Obes. 2015;39(7):1070-8.

17. Ogden CL, Carroll MD, Fakhouri TH, Hales CM, Fryar CD, Li X, et al. Prevalence of obesity among youths by household income and education level of head of household - United States 2011-2014. Morb Mortal Wkly Rep. 2018;67(6):186-9. 
18. Jackson SL, Yang EC, Zhang Z. Income disparities and cardiovascular risk factors among adolescents. Pediatrics. 2018;142(5):1.

19. National Center for Health Statistics, Centers for Disease Control and Prevention. National health and nutrition examination survey. 2017 [cited 2020 Jan 10]. Available from: https://www.cdc.gov/nchs/nhanes/about_nha nes.htm

20. Centers for Disease Control and Prevention. National health and nutrition examination survey (NHANES) anthropometry procedures manual. 2016.

21. Li C, Ford ES, Mokdad AH, Cook S. Recent trends in waist circumference and waist-height ratio among US children and adolescents. Pediatrics. 2006; 118(5):e1390.

22. Murakami K, MBE L. Meal and snack frequency in relation to diet quality in US children and adolescents: the National Health and Nutrition Examination Survey 2003-2012. Public Health Nutr. 2016;19(09):1635-44 [cited 2019 Jan 8]. Available from: http://www.journals.cambridge.org/abstract_S136898001 6000069.

23. Johnson CL, Paulose-Ram R, Ogden CL. National Health and Nutrition Examination Survey: Analytic Guidelines, 1999-2010. National Center for Health Statistics. Vital Health Stat. 2014;161:1 Available from: https://www. cdc.gov/nchs/data/series/sr_02/sr02_161.pdf.

24. Centers for Disease Control and Prevention, National Center for Health Statistics. National Health and Nutrition Examination Survey: Analytic Guidelines, 2011-2014 and 2015-2016: National Center for Health Statistics; 2018. Available from: https://wwwn.cdc.gov/nchs/nhanes/ana lyticguidelines.aspx\#analytic-guidelines

25. He Q, Horlick M, Thornton J, Wang J, Pierson RN, Heshka S, et al. Sexspecific fat distribution is not linear across pubertal groups in a multiethnic study. Obes Res. 2004;12(4):725-33.

26. Abbassi V. Growth and normal puberty. Pediatrics. 1998;102(2 III):507-11.

27. Brambilla P, Bedogni G, Heo M, Pietrobelli A. Waist circumference-to-height ratio predicts adiposity better than body mass index in children and adolescents. Int J Obes. 2013;37(7):943-6.

28. Hall DMB, Cole TJ. What use is the BMI? Arch Dis Child. 2006;91(4):283-6.

29. Deurenberg P, Yap M, Van Staveren W. Body mass index and percent body fat. Int J Obes. 1998;22:1164-71.

30. Okosun IS, Boltri JM, Eriksen MP, Hepburn VA. Trends in Abdominal Obesity in Young People: United States 1988-2002. Ethn Dis. 2006;16:338-44 Available from: https://www.ethndis.org/priorarchives/ethn-16-02-338.pdf.

31. Tybor DJ, Lichtenstein AH, Dallal GE, Daniels SR, Must A. Racial differences in central adiposity in a longitudinal cohort of black and white adolescent females. BMC Pediatr. 2010;10:1

32. Costa de Oliveira Forkert E, de Moraes ACF, Carvalho HB, Kafatos A, Manios $Y$, Sjöström M, et al. Abdominal obesity and its association with socioeconomic factors among adolescents from different living environments. Pediatr Obes. 2017;12(2):110-9.

33. Hiza HAB, Casavale KO, Guenther PM, Davis CA. Diet quality of Americans differs by age, sex, race/ethnicity, income, and education level. J Acad Nutr Diet. 2013;113(2):297-306. https://doi.org/10.1016/j.jand.2012.08.011.

34. Ali MK, Bullard KM, Beckles GL, Stevens MR, Barker L, Narayan KMV, et al. Household income and cardiovascular disease risks in U.S. children and young adults: analyses from NHANES 1999-2008. Diabetes Care. 2011;34(9): 1998-2004 [cited 2019 Sep 12]. Available from: http://www.ncbi.nlm.nih. gov/pubmed/21868776.

35. Herd P, Goesling B, House JS. Socioeconomic position and health: the differential effects of education versus income on the onset versus progression of health problems. J Health Soc Behav. 2007;48(3):223-38.

36. Sallis JF, Slymen DJ, Conway TL, Frank LD, Saelens BE, Cain K, et al. Income disparities in perceived neighborhood built and social environment attributes. Health Place. 2011;17(6):1274-83. https://doi.org/10.1016/j.hea Ithplace.2011.02.006.

37. Larson NI, Story MT, Nelson MC. Neighborhood Environments. Disparities in Access to Healthy Foods in the U.S. Am J Prev Med. 2009;36(1):74-81.e10. https://doi.org/10.1016/j.amepre.2008.09.025

38. Juonala M, Harcourt BE, Saner C, Sethi M, Saffery R, Magnussen CG, et al. Neighbourhood socioeconomic circumstances, adiposity and cardiometabolic risk measures in children with severe obesity. Obes Res Clin Pract. 2019;13(4):345-51. https://doi.org/10.1016/j.orcp.2019.05.004.

39. Xu F, Cohen SA, Greaney ML, Greene GW. The association between US adolescents' weight status, weight perception, weight satisfaction, and their physical activity and dietary behaviors. Int J Environ Res Public Health. 2018; 15(9):1931.
40. Centers for Disease Control and Prevention, National Center for Health Statistics. 2015-2016 Data Documentation, Codebook, and Frequencies Demographic Variables and Sample Weights (DEMO_I): National Health and Nutrition Examination Survey; 2017. [cited 2020 Jan 10]. Available from: https://wwwn.cdc.gov/Nchs/Nhanes/2015-2016/DEMO_I.htm

41. Frederick CB, Snellman K, Putnam RD. Increasing socioeconomic disparities in adolescent obesity. Proc Natl Acad Sci U S A. 2014;111(4):1338-42.

42. Hales CM, Carroll MD, Fryar CD, Ogden CL. Prevalence of obesity among adults and youth: United States, 2015-2016, vol. 288. Hyattsville: NCHS data brief; 2017. Available from: https://www.cdc.gov/nchs/data/databriefs/db2 88.pdf

43. Oates $G R$, Jackson BE, Partridge EE, Singh KP, Fouad MN, Bae S. Sociodemographic patterns of chronic disease: how the mid-south region compares to the rest of the country. Am J Prev Med [Internet]. 2017;52(1): S31-9 Available from: https://linkinghub.elsevier.com/retrieve/pii/S07493 79716304020

44. Quiñones AR, Botoseneanu A, Markwardt S, Nagel CL, Newsom JT, Dorr DA, et al. Racial/ethnic differences in multimorbidity development and chronic disease accumulation for middle-aged adults. PLoS One. 2019;14(6):1-13.

45. CDC. NHANES Questionnaires, Datasets, and Related Documentation. National Health and Nutrition Examination Survey. 2020 [cited 2020 Oct 12]. Available from: https://wwwn.cdc.gov/nchs/nhanes/default.aspx

\section{Publisher's Note}

Springer Nature remains neutral with regard to jurisdictional claims in published maps and institutional affiliations.
Ready to submit your research? Choose BMC and benefit from:

- fast, convenient online submission

- thorough peer review by experienced researchers in your field

- rapid publication on acceptance

- support for research data, including large and complex data types

- gold Open Access which fosters wider collaboration and increased citations

- maximum visibility for your research: over $100 \mathrm{M}$ website views per year

At BMC, research is always in progress.

Learn more biomedcentral.com/submissions 